\title{
Transposon Insertion in the ftsK Gene Impairs In Planta Growth and Lesion-Forming Abilities in Pseudomonas syringae pv. syringae B728a
}

\author{
Thomas G. Kinscherf, ${ }^{1}$ Susan S. Hirano, ${ }^{1}$ and David K. Willis ${ }^{2}$ \\ ${ }^{1}$ Department of Plant Pathology, University of Wisconsin-Madison, 1630 Linden Drive, Madison 53706, \\ U.S.A.; ${ }^{2}$ U.S. Department of Agriculture, Agricultural Research Service, University of Wisconsin-Madison, \\ 1630 Linden Drive, Madison 53706, U.S.A. \\ Accepted 20 July 2000.
}

\begin{abstract}
A Tn5 insertion in the ftsK gene of Pseudomonas syringae pv. syringae B728a impaired brown spot lesion formation on Phaseolus vulgaris, the ability to grow within bean leaves, and swarming ability on semisolid agar. Plasmids containing the $f$ ts $K$ gene were sufficient to complement the original Tn5 mutant for lesion formation and swarming and partially restored in planta growth.
\end{abstract}

Additional keywords: pathogenicity, plant pathogen, virulence.

Pseudomonas syringae pv. syringae is the causal agent of bacterial brown spot on snap bean (Phaseolus vulgaris L.). We have previously identified a number of lesion-minus mutants in a screen of 6,401 Tn5 mutants of B728a (Rich and Willis 1997). One of these mutants, KW3815, was of particular interest because it retained the ability to produce syringomycin and extracellular protease, unlike mutants with defects in gacS, gacA, or salA (Kinscherf and Willis 1999; Kitten et al. 1998). Furthermore, KW3815 was unaffected in its ability to cause a hypersensitive response in tobacco. KW3815 Tn5 and the flanking chromosomal DNA was previously isolated as a cosmid (pJJR3815-Tn5 H) and used to demonstrate that Tn5 insertion is causal to the mutant's lesion-minus phenotype by site-specific recombination (Rich and Willis 1997). To identify the affected locus, an approximately 9.5-kb EcoRI fragment containing the transposon was isolated from pJJR3815-Tn5 $\mathrm{H}$ and cloned into pBluescriptKS+ to yield pKSEcoR1. The EcoRI fragment was isolated from pKSEcoRI and used to probe a cosmid library of wild-type B728a chromosomal DNA by colony blotting. A hybridizing cosmid (pWT3B) that restored lesion-forming ability to KW3815 was found. A 3.6-kb HindIII fragment of pWT3B, cloned into pLAFR3 in pLAFR-H3 and pLAFR-H7 orientations, restored lesion-forming ability to KW3815 (data not shown). The DNA sequence of a Bam $\mathrm{HI}$ fragment isolated

Corresponding author: D. K. Willis; Telephone: +1-608-262-5063; Fax: +1-608-263-2626; E-mail: dkwillis@facstaff.wisc.edu

This article is in the public domain and not copyrightable. It may be freely reprinted with customary crediting of the source. The American Phytopathological Society, 2000. from pKSEcoR 1 and cloned into pBluescriptKS+ identified the Tn5 site of insertion in a fts $K$ sequence analog, a gene described in Escherichia coli as being involved in cell division (Begg et al. 1995).

Further sequencing was performed with a restoring 3.6-kb HindIII fragment isolated from pWT3B cloned into pBluescript$\mathrm{KS}+$ as template. This HindIII fragment contains the entire ftsK open reading frame (ORF) but only the amino termini of the aat and lolA genes, indicating that the fts $K$ gene complements the mutant phenotypes of KW3815. The FtsK protein predicted by the B728a DNA sequence is approximately $88 \mathrm{kDa}$, far smaller than the previously described $E$. coli protein $(147 \mathrm{kDa})$, yet larger than other FtsK proteins in the database (Fig. 1A). Figure $1 \mathrm{~B}$ shows the genetic organization of the B728a chromosome in the region surrounding the $3815 \mathrm{Tn} 5$ insertion The gene order is aat-ftsK-lolA-ORF1-ORF2-serS. The first gene, aat, codes for leucyl, phenylalanyl-tRNA-protein transferase (Shrader et al. 1993), an enzyme involved in $\mathrm{N}$-end rule regulation The lolA gene, an analog of a gene that in $E$. coli encodes a periplasmic protein chaperone for lipoproteins, is in a different reading frame than ftsK. ORF1 begins immediately after the lolA stop codon and is in the same reading frame. Database analyses indicate a very high degree of conservation for analogs of this gene in prokaryotes and eukaryotes. The gene order ftsK-lolA-ORF1 is conserved in Haemophilus influenza and E. coli as well as B728a, suggesting that this gene cluster might be a general feature in gram-negative bacteria. ORF2 appears to be an analog of potential bacterial membrane proteins of unknown function. The last gene defined in the region is serS, the gene for serine tRNA synthetase. The sequence predicts that $f t s K$, lolA, ORF1, ORF2, and serS are transcribed in the same direction.

As initially described by Rich and Willis (1997), colonies of KW3815 on agar media were more mucoid than those of wildtype B728a. This was most pronounced on nutrient agar supplemented with glycerol (data not shown). Microscopy of KW3815 revealed unusual chains of cells, with joints between individual cells often being strangely kinked out of plane relative to the wild type (data not shown). The proportion of cells in these chains varied from experiment to experiment, with many "single" cells (actually doublet cells typical of a growing culture of B728a) present in each culture, indicating a 
potential conditional phenotype. Remarkably, the chain formation had almost no effect on the ability of KW3815 to grow in rich media based on turbidity measurements (data not shown). Filamentation of the type found with the original ftsK mutant in E. coli (Begg et al. 1995) was not observed with KW3815 (data not shown). Chain formation rather than filamentation is consistent with the location of the transposon insertion being in the carboxy-terminal half of fts $K$ (domain 3) (Recchia et al. 1999).

KW3815 was deficient for a previously described agar surface motility phenotype found in B728a (Kinscherf and Willis 1999) with the strain usually growing as a roughly circular colony in the center of the plate until becoming mucoid after
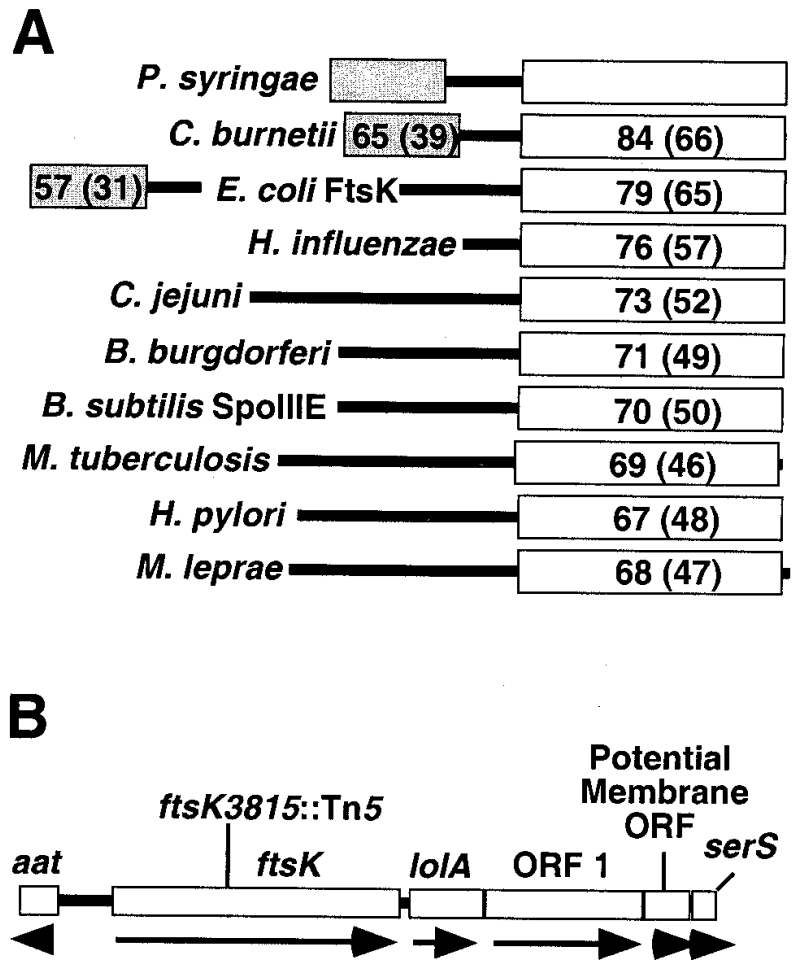

Fig. 1. A, Schematic representation of amino acid similarities (identities) of the Pseudomonas syringae pv. syringae FtsK open reading frame (ORF) compared with members of the FtsK/SpoIIIE protein family from various bacteria. Protein sequences from bacteria were individually compared with $P$. syringae pv. syringae FtsK protein by the BestFit program, version 8 (Genetics Computer Group, Madison, WI, U.S.A.). Open boxes $=$ conserved $\mathrm{C}$-terminal domain common among all members of this protein family. Filled boxes $=\mathrm{N}$-terminal region common to a few members of the protein family. Thick black line $=$ Regions without significant similarity. Percentage of amino acid similarities (identities) for the entire FtsK/SpoIIIE ORF of various proteins when compared with $P$. syringae pv. syringae FtsK ORF were Coxiella burnetii (Accession No. P39920), 74(54); Escherichia coli (Accession No. Z49932), 69(51); Haemophilus influenza (Accession No. P45264), 73(54); Campylobacter jejuni (Accession No. Q46089), 59(37); Borrelia burgdorferi (Accession No. AE001136), 61(37); Bacillus subtilis (Accession No. P21458), 60(38); Mycobacterium tuberculosis (Accession No. AL008967), 59(38); M. leprae (Accession No. Z94723), 60(37); and Helicobacter pylori (Accession No. B64656), 60(39). B, Gene order within $P$. syringae pv. syringae fts $K$ chromosomal region. Open boxes $=$ predicted ORF. Arrows $=$ direction of transcription predicted from DNA sequence. Thick black lines = intergenic DNA regions. The position of ftsK3815::Tn5 insertion at nucleotide 1378 within the $f t s K$ gene is indicated. several days of incubation (data not shown). This "swarming" deficiency was generally restored in the mutant by the HindIII subclone.

We have previously demonstrated an absolute deficiency for swarming in gacS and gacA mutants of B728a (Kinscherf and Willis 1999). The swarming phenotype in the fts $K$ mutant was more variable than that observed for gac mutants, with some aberrant surface spreading occurring in isolated instances (data not shown). The significance of the ftsK phenotype is difficult to estimate because KW3815 is not defective for motility by microscopy (data not shown).

The necrotic reaction apparent in the wild type did not develop with KW3815 when inoculated into growth chambergrown bean leaves at approximately $10^{6} \mathrm{CFU} / \mathrm{ml}$ (Rich and Willis 1997). In leaf infiltration growth assays, maximum population sizes attained by the mutant were approximately 2.5 orders of magnitude less than the wild type (Fig. 2). Results were similar in three independent experiments. The absolute numbers should be considered advisory, however, given that the multicellular chain formation mentioned below might affect accurate determination of colony-forming units relative to wild type. Additionally, the available microscopic evidence suggests that the effects of such chain formation on colonyforming units determination, if any, would be arithmetic rather than logarithmic. Expression of the fts $K$ gene in trans only partially restored growth in planta to KW3815 (Fig. 2) This partial restoration was apparently not due to simple plasmid instability because population sizes determined with and without selection for the plasmid-borne $\mathrm{Tet}^{\mathrm{r}}$ marker were essentially the same after 5 days of growth (Fig. 2).

Our results indicate that the transposon insertion, which was causal to the loss of pathogenicity in KW3815, is in the ftsK gene analog of B728a. KW3815 illustrates the difficulty of assigning a role to lesion formation in a gene such

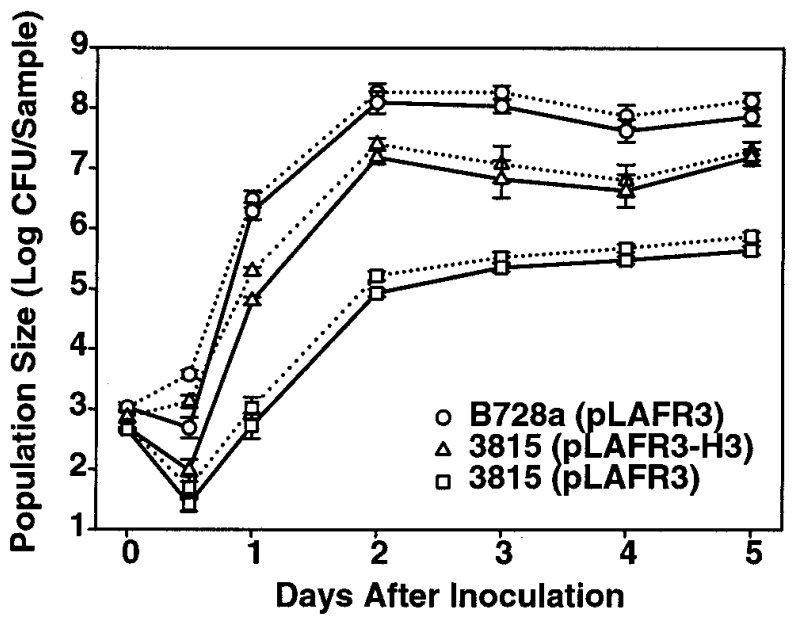

Fig. 2. Mean population sizes of Pseudomonas syringae pv. syringae B728a(pLAFR3), KW3815(pLAFR3), and KW3815(pLAFR3-H3) as a function of days after inoculation. Bacterial suspensions of approximately $1 \times 10^{6} \mathrm{CFU} / \mathrm{ml}$ were infiltrated into primary leaves of snap bean cv. Bush Blue Lake 274. Each datum point represents mean bacterial population and standard error based on five 6-mm leaf disks from five different leaves at each time point. The population size was determined with (solid line) and without (dotted line) selection for the $\operatorname{Tet}^{\mathrm{r}}$ marker of vector pLAFR3. 
as $f t s K$ because it also affects growth in the plant environment. In a pathogen such as $P$. syringae pv. syringae in which growth in association with leaves is necessary for disease development, any mutation that affects growth in planta may also affect lesion formation (Hirano and Upper 1990). Restoration of lesion formation to KW3815 with only partial restoration of in planta growth hints at a minimum threshold population size capable of disease manifestation, at least in the artificial world of the laboratory. Studies with strains containing mutations in gacS, gacA, or salA demonstrated that bacterial growth within bean leaves does not lead to macroscopic lesions (Kitten et al. 1998; Rich et al. 1994; Willis et al. 1990). It seems clear that metabolic processes, in addition to bacterial multiplication, are required for symptom development on beans. It does seems likely, however, that the lesion-minus phenotype of KW3815 is due to its poor growth on beans rather than as a result of direct involvement of $f t s K$ in lesion-specific processes.

\section{ACKNOWLEDGMENTS}

We thank L. Hogan and J. McEvoy for their helpful comments on the manuscript. We also thank R. Spear for help with microscopy. This work was supported by NSF grant MCB-9419023 presented to D. K. Willis.

\section{LITERATURE CITED}

Begg, K. J., Dewar, S. J., and Donachie, W. D. 1995. A new Escherichia coli cell division gene, ftsK. J. Bacteriol. 177:6211-6222.

Hirano, S. S., and Upper, C. D. 1990. Population biology and epidemiology of Pseudomonas syringae. Annu. Rev. Phytopathol 28:155-177.

Kinscherf, T. G., and Willis, D. K. 1999. Swarming by Pseudomonas syringae $\mathrm{B} 728$ a requires $\operatorname{gacS}(\operatorname{lemA})$ and gacA but not the acylhomoserine lactone gene ahlI. J. Bacteriol. 181:4133-4136.

Kitten, T., Kinscherf, T. G., McEvoy, J. L., and Willis, D. K. 1998. A newly identified regulator is required for virulence and toxin production in Pseudomonas syringae. Mol. Microbiol. 28:917-930.

Recchia, G. D., Aroyo, M., Wolf, D., Blakely, G., and Sherratt, D. J. 1999. FtsK-dependent and -independent pathways of Xer site-specific recombination. EMBO J. 18:5724-5734.

Rich, J. J., Kinscherf, T. G., Kitten, T., and Willis, D. K. 1994. Genetic evidence that the gacA gene encodes the cognate response regulator for the lemA sensor in Pseudomonas syringae. J. Bacteriol. 176:7468-7475.

Rich, J. J., and Willis, D. K. 1997. Multiple loci of Pseudomonas syringae pv. syringae are involved in pathogenicity on bean: Restoration of one lesion deficient mutant requires two tRNA genes. J. Bacteriol. 179:2247-2258.

Shrader, T. E., Tobias, J. W., and Varshavsky, A. 1993. The N-end rule in Escherichia coli: Cloning and analysis of the leucyl, phenylalanyltRNA-protein transferase gene aat. J. Bacteriol. 175:4364-4374.

Willis, D. K., Hrabak, E. M., Rich, J. J., Barta, T. M., Lindow, S. E., and Panopoulos, N. J. 1990. Isolation and characterization of a Pseudomonas syringae pv. syringae mutant deficient in lesion formation on bean. Mol. Plant-Microbe Interact. 3:149-156. 